\title{
Oxytocin receptor ligand binding in embryonic tissue and postnatal brain development of the C57BL/6J mouse
}

\author{
Elizabeth A. D. Hammock ${ }^{1 *}$ and Pat Levitt ${ }^{2 *}$ \\ 1 Vanderbilt Kennedy Center and Department of Pediatrics, Vanderbilt University School of Medicine, Vanderbilt University, Nashville TN, USA \\ ${ }^{2}$ Department of Pediatrics, Children's Hospital Los Angeles and Keck School of Medicine of the University of Southern California, Los Angeles, CA, USA
}

\author{
Edited by: \\ Alaine Keebaugh, Emory University, \\ USA \\ Reviewed by: \\ Charles W. Wilkinson, University of \\ Washington, USA \\ Ron Stoop, University of Lausanne, \\ Switzerland

\section{${ }^{*}$ Correspondence:} \\ Elizabeth A. D. Hammock, \\ Department of Pediatrics, Vanderbilt \\ University, 2215 Garland Ave., Light \\ Hall, Rm 1115, Nashville, \\ TN 37232, USA \\ e-mail: liz.hammock@vanderbilt.edu; \\ Pat Levitt, Department of Pediatrics, \\ Children's Hospital Los Angeles, \\ 4650 Sunset BIvd., Los Angeles, \\ CA 90027, USA \\ e-mail: plevitt@med.usc.edu
}

Oxytocin (OXT) has drawn increasing attention as a developmentally relevant neuropeptide given its role in the brain regulation of social behavior. It has been suggested that OXT plays an important role in the infant brain during caregiver attachment in nurturing familial contexts, but there is incomplete experimental evidence. Mouse models of OXT system genes have been particularly informative for the role of the OXT system in social behavior, however, the developing brain areas that could respond to ligand activation of the OXT receptor (OXTR) have yet to be identified in this species. Here we report new data revealing dynamic ligand-binding distribution of OXTR in the developing mouse brain. Using male and female C57BL/6J mice at postnatal days (P) 0, 7, 14, 21, 35, and 60 we quantified OXTR ligand binding in several brain areas which changed across development. Further, we describe OXTR ligand binding in select tissues of the near-term whole embryo at E18.5. Together, these data aid in the interpretation of findings in mouse models of the OXT system and generate new testable hypotheses for developmental roles for OXT in mammalian systems. We discuss our findings in the context of developmental disorders (including autism), attachment biology, and infant physiological regulation.

Summary: Quantitative mapping of selective OXTR ligand binding during postnatal development in the mouse reveals an unexpected, transient expression in layers II/III throughout the mouse neocortex. OXTR are also identified in several tissues in the whole late embryo, including the adrenal glands, brown adipose tissue, and the oronasal cavity.

Keywords: oxytocin, adrenal gland, kidney, oronasal cavity, autism, autoradiography, experience-dependent plasticity, neocortex

\section{INTRODUCTION}

There is substantial experimental evidence in humans and preclinical models that oxytocin (OXT) plays a role in adult behavior. In humans, whereas data from genetic association studies are difficult to replicate (Bakermans-Kranenburg and van Ijzendoorn, 2013), results from studies with intranasal treatment with OXT are consistent with a role for OXT in adult human social behavior (Guastella and Macleod, 2012). In mice in which Oxt has been constitutively deleted, adults exhibit poor social recognition behavior (Ferguson et al., 2000; Macbeth et al., 2009). Similarly, adult OXT receptor (Oxtr) knock-out mice also display evidence of poor social recognition behavior (Takayanagi et al., 2005; Lee et al., 2008a; Macbeth et al., 2009). OXTR signaling seems to play more of role in intrastrain social recognition, which is a more difficult perceptual and memory task than interstrain social recognition (Macbeth et al., 2009). In addition, the adult Oxtr KO mouse is seizure-prone and has poor reversal learning, a laboratory proxy of poor cognitive flexibility (Sala et al., 2011). Maternal care is partially impaired in both lines of mice (Takayanagi et al., 2005; Pedersen et al., 2006).

Hypotheses of the underlying mechanisms that generate these behavioral phenotypes in mutant mice, in which target genes are deleted constitutively from all tissues, have been informed mostly by our understanding of OXTR ligand binding distribution in the adult mouse brain. For example, the septum, the hippocampus, the amygdala, and the piriform cortex have all been implicated. The possibility also exists that congenital OXTR loss throughout mouse development makes a significant contribution to reported disruption of adult behaviors, as has been shown for the serotonin (5-HT) 1a receptor (Gross et al., 2002). To selectively ascertain a role for OXTR in the adult brain, Lee et al generated a floxed Oxtr mouse, which has been used to delete Oxtr from selective brain areas in the adult by introduction of a cell type specific Cre recombinase (Lee et al., 2008a,b; Macbeth et al., 2009; Pagani et al., 2011). Despite a growing number of studies in mouse genetic models (Table 1), distribution data regarding the contribution of OXTR signaling during experience-dependent development has not been established in the mouse, but instead has been guided by developmental expression patterns obtained in rats (Shapiro and Insel, 1989; Snijdewint et al., 1989; Tribollet et al., 1989) and voles (Wang and Young, 1997), which differ from each other. Tritiated oxytocin and ${ }^{125}$ I-labeled selective antagonist mapping of developing rodent brains demonstrate robust receptor presence in postnatal development (Tribollet et al., 1989, 1991, 1992; Wang and Young, 1997). Some brain areas show dense ligand binding during postnatal development with very little expression in adults. While the location of the receptor binding varies with species, rats and voles have a peak of OXTR binding in some brain 
Table 1 | Mouse models of oxytocin system function.

\begin{tabular}{|c|c|c|c|}
\hline Gene & Mouse name & Description of manipulation & Original reference \\
\hline Oxt & OXT KO (OT KO); Oxt ${ }^{t m 1 Z u k}$ & $\begin{array}{l}\text { Congenital knockout on mixed 129/B6; * also available on } \\
\text { C57Bl/6j }\end{array}$ & $\begin{array}{l}\text { Nishimori et al., 1996; *Hammock } \\
\text { and Levitt, unpublished }\end{array}$ \\
\hline Oxt & OXT KO; Oxt $t^{\operatorname{tm} 1 W_{s y}}$ & Congenital knockout on mixed 129/B6 & Young et al., 1996b \\
\hline$O x t$ & Oxt/EGFP & $\begin{array}{l}\text { EGFP labeled OXT-neurophysin pre-prohormone; } \\
\text { backcrossed for many generations to C57BL/6 }\end{array}$ & Zhang et al., 2002 \\
\hline Oxtr & OXTR KO; Oxtrtm1.1Knis & $\begin{array}{l}\text { Congenital knockout on mixed 129/B6; *also available on } \\
\text { C57Bl/6j }\end{array}$ & $\begin{array}{l}\text { Takayanagi et al., 2005; * This } \\
\text { report }\end{array}$ \\
\hline Oxtr & $O x \operatorname{tr}^{\operatorname{tm} 1(K O M P)} \mathrm{Vlcg}$ & Congenital knockout on C57BL/6 & Velocigene; www.komp.org \\
\hline Oxtr & Oxtrflox; Oxtrtm1.1Wsy & $\begin{array}{l}\text { Floxed OXTR for use with Cre recombinase for selective } \\
\text { excision }\end{array}$ & Lee et al., 2008a \\
\hline Oxtr & Oxtr-Venus & $\begin{array}{l}\text { Venus reporter in place of Oxtr; reporter positive mice are } \\
\text { Oxtr hemizygous or KO; on mixed 129/B6 }\end{array}$ & Yoshida et al., 2009 \\
\hline Oxtr & Oxtr-EGFP; Tg(Oxtr-EGFP)GO185Gsat & $\begin{array}{l}\text { BAC transgenic with OXTR promoter driven expression of } \\
\text { EGFP; theoretically does not interfere with native OXTR }\end{array}$ & GENSAT, 2003; Gong et al., 2003 \\
\hline Oxtr & OXTR-cre; Tg(Oxtr-cre)ON66Gsat & Cre recombinase driven by the OXTR promoter & GENSAT, 2003 \\
\hline Oxtr & tetOXTR & $\begin{array}{l}\text { Tetracycline responsive OXTR promoter for selective } \\
\text { regional and/or temporal over-expression; backcrossed to } \\
\text { C57BI/6j }\end{array}$ & L. J. Muglia pers. comm \\
\hline Oxtr & OTR-LacZ & $\begin{array}{l}\text { Oxtr promoter drives } L a c Z \text { expression; LacZ allele results in } \\
\text { lower Oxtr expression; on } 129 \times \text { Balb/c mixed background }\end{array}$ & Gould and Zingg, 2003 \\
\hline$C D 38$ & $\mathrm{CD}^{3} 8^{-/-}$ & $\begin{array}{l}\text { Congenital loss of CD38 which regulates secretion of OXT; } \\
\text { On ICR x B6 X DBA }\end{array}$ & Kato et al., 1999; Jin et al., 2007 \\
\hline
\end{tabular}

areas in the 2nd and 3rd postnatal weeks. The species diversity in developmental OXTR distribution raises the question of the similarities and differences in the characteristics of OXTR expression patterns in the developing mouse brain compared to other species.

Because of observed species diversity in OXTR expression patterns and because OXTRs have not been mapped developmentally in mice, we questioned the potential role for M. musculus-specific developmental expression patterns that might better inform hypotheses of behavior in mouse genetic models (Table 1) in which OXTR signaling is altered genetically either directly, or indirectly through ligand alteration.

In this report, we qualitatively map and quantitate developmental expression of OXTR ligand binding in the postnatal C57BL/6J mouse brain. We also identify OXTR binding in the near term mouse embryo (E18.5) to identify potential OXT sites of action in the whole organism in the transition to postnatal life.

\section{MATERIALS AND METHODS \\ MICE}

Mice used in this study were bred in our animal facility from adult C57BL/6J mice obtained from Jackson Laboratories (Bar Harbor, ME). All procedures were performed after approval by the Institutional Animal Care and Use Committee of Vanderbilt University in accordance with state and federal guidelines. Timed pregnancies were generated, and females were checked daily for litters. The first morning appearance of a litter was noted as postnatal day 0 (P0). Pre-weaning litters were harvested on P0, P7, and P14. Individual litters each contributed to one time point only. Two litters were harvested immediately after weaning at P21, and individuals from several more litters were harvested at P35 and P60. E18.5 embryos were harvested from a timed-pregnant C57BL/6J mouse purchased from Jackson Laboratories and gestational age was confirmed by crown-rump length $(20 \pm 0.5 \mathrm{~mm})$. Oxtr mice (Oxtr $\left.{ }^{\text {tm1.1Knis }}\right)$ were a generous gift from Larry Young (Emory University). We backcrossed these mice to C57BL/6J and confirmed achieving congenic status with strain specific markers (Speed Congenics Service, Jackson Laboratories, Bar Harbor, $\mathrm{ME})$.

\section{SEX DETERMINATION}

Sex determination of mice aged P14 and older was performed by an experienced rater (Elizabeth A. D. Hammock) by anogenital distance. Embryonic mice and mice aged P0 and P7 were genotyped to determine genetic sex using established methods (Jimenez et al., 2003). The forward primer $\left(5^{\prime}\right.$-ccgctgccaaattctttgg- $\left.3^{\prime}\right)$ and the reverse primer $\left(5^{\prime}\right.$ tgaagcttttggctttgag- $3^{\prime}$ ) generate a 290 bp product from the Smcy gene on the $\mathrm{Y}$ chromosome, and a $330 \mathrm{bp}$ product from the Smcx homolog on the $\mathrm{X}$ chromosome under the following thermal cycling conditions: $95^{\circ} \mathrm{C}$ for $7 \mathrm{~min} ; 35$ cycles of $93^{\circ} \mathrm{C}$ for $30 \mathrm{~s}$, $58^{\circ} \mathrm{C}$ for $30 \mathrm{~s}, 72^{\circ} \mathrm{C}$ for $30 \mathrm{~s} ; 72^{\circ} \mathrm{C}$ for $10 \mathrm{~min}$.

\section{RECEPTOR AUTORADIOGRAPHY}

Each age for analyses of OXTR ligand binding consisted of animals from at least two separate litters. For the quantitative binding study, a total of 3 males and 3 females for each age were used. For the post-natal experiments, the autoradiography was performed 3 separate times with each age and sex represented. E18.5 whole embryos were harvested for examining tissue patterns of OXTR ligand binding. Brains (or embryos) were frozen in powdered dry ice and stored at $-80^{\circ} \mathrm{C}$ until cryosectioning. 
Tissue was cut at $20 \mu \mathrm{m}$ in 6 series and thaw mounted onto Superfrost Plus slides. Sections were stored at $-80^{\circ} \mathrm{C}$ until used in the receptor autoradiography protocol. Receptor autoradiography was performed exactly as described previously (Hammock and Levitt, 2012) on rostro-caudal series of sections with $50 \mathrm{pM}$ of selective ${ }^{125} \mathrm{I}$ OXTR ligand: ornithine vasotocin analog (vasotocin, $\mathrm{d}\left(\mathrm{CH}_{2}\right)_{5}\left[\mathrm{Tyr}(\mathrm{Me})^{2}, \mathrm{Thr}^{4}, \mathrm{Orn}^{8},{ }^{125} \mathrm{I}\right] \mathrm{Tyr}^{9}$ $\left.\mathrm{NH}_{2}\right]$; ([$\left.{ }^{125} \mathrm{I}\right]$-OVTA, NEX254, Perkin-Elmer, Inc., Boston, MA). Autoradiographic films (Kodak Biomax MR film, Carestream Health, Inc., Rochester, NY, USA) were developed after a $70 \mathrm{~h}$ co-exposure with ${ }^{14} \mathrm{C}$ autoradiographic standards (American Radiolabeled Chemicals, St. Louis, MO, USA).

\section{IMAGE ANALYSIS}

After receptor autoradiography, slides were post-processed for acetylcholinesterase (Lim et al., 2004) and/or Nissl staining following standard protocols (Catania et al., 2008). Autoradiographic films and processed slides were scanned at 1200 dpi at 8-bit with a high resolution flatbed scanner (Epson Perfection V600, Suwa, Japan) and regions of interest were identified by comparison of the film image to landmarks in the post-processed slides. Image measurements were obtained in Image (NIH, Bethesda, MD) from three consecutive sections with hand selected ROI and analyzed by interpolation "interp1," Matlab 7.0.4 (TheMathworks, Natick, MA, USA) to the linear range of the ${ }^{14} \mathrm{C}$ autoradiographic standard on the same film (Miller and Zahniser, 1987). Tissue background values were obtained from the dorsal striatum of each sample and subtracted from the region specific data to generate net values reflected in the graphed data. This resulted in average values of $0 \mu \mathrm{Ci} / g$ for each quantified brain area in Oxtr KO. For quantification, no adjustments were made to the images other than image inversion. Composite images for figures were created with the TurboReg (Thevenaz et al., 1998) plug-in for ImageJ using the rigid body alignment algorithm. For pseudocolor composites, the autoradiography images were adjusted for brightness to minimize the appearance of the film background.

\section{RESULTS}

As previously established, the ligand ([$\left.{ }^{125} \mathrm{I}\right]$-OVTA) used in this report is highly selective for OXTR in mice, rats, and voles as determined by displacement with a competitive unlabeled ligand (Elands et al., 1988; Insel and Shapiro, 1992; Insel et al., 1993), and by absence of specific binding in the OXTR KO mouse (Figure 1) above tissue background, as others have shown (Takayanagi et al., 2005; Lee et al., 2008a). We observed ligand binding (Figure 1) in several brain regions previously reported for high OXTR ligand binding in adult mice (Insel et al., 1993). For example, the OXTR ligand binding was evident in the lateral septum, diagonal band, piriform cortex, the central and medial amygdala, the hypothalamus, and CA3 of the hippocampus. By comparison to post-processed neuroanatomical landmarks and a neuroanatomical atlas (Paxinos and Franklin, 2001) (Allen Brain Atlas), we confirmed binding in the following areas (Figure 1) at most ages examined: accessory and main olfactory bulbs, claustrum, endopiriform cortex, bed nucleus of the stria terminalis, ventral caudatoputamen, and the periventricular thalamus. We also identified a developmental peak of ligand binding in the neocortex, evident in pre-weaning mice (Figures 1, 2).

To ascertain developmental shifts in receptor density, we quantified several brain areas that exhibited unique patterns of binding (Figure 2): the hippocampus, the lateral septum, and the neocortex. Each brain area was analyzed by Two-Way ANOVA for sex and age. The hippocampus measured dorsally at CA3 showed no main effect of sex $\left[F_{(1,24)}=0.73, p=0.40\right]$, a significant main effect of age $\left[F_{(5,24)}=7.93, p<0.001\right]$, and no sex $\times$ age interaction $\left[F_{(5,24)}=0.41, p=0.84\right]$. The septum showed a trend for a main effect of sex $\left[F_{(1,24)}=3.34, p=0.08\right]$, a main effect of age $\left[F_{(5,24)}=7.87, p<0.001\right]$, and no sex $\times$ age interaction $\left[F_{(5,24)}=0.75, p=0.59\right]$. OXTR density captured throughout all layers of S1 neocortex indicates no main effect of sex $\left[F_{(1,24)}=2.31, p=0.14\right]$, a main effect of age $\left[F_{(5,24)}=\right.$

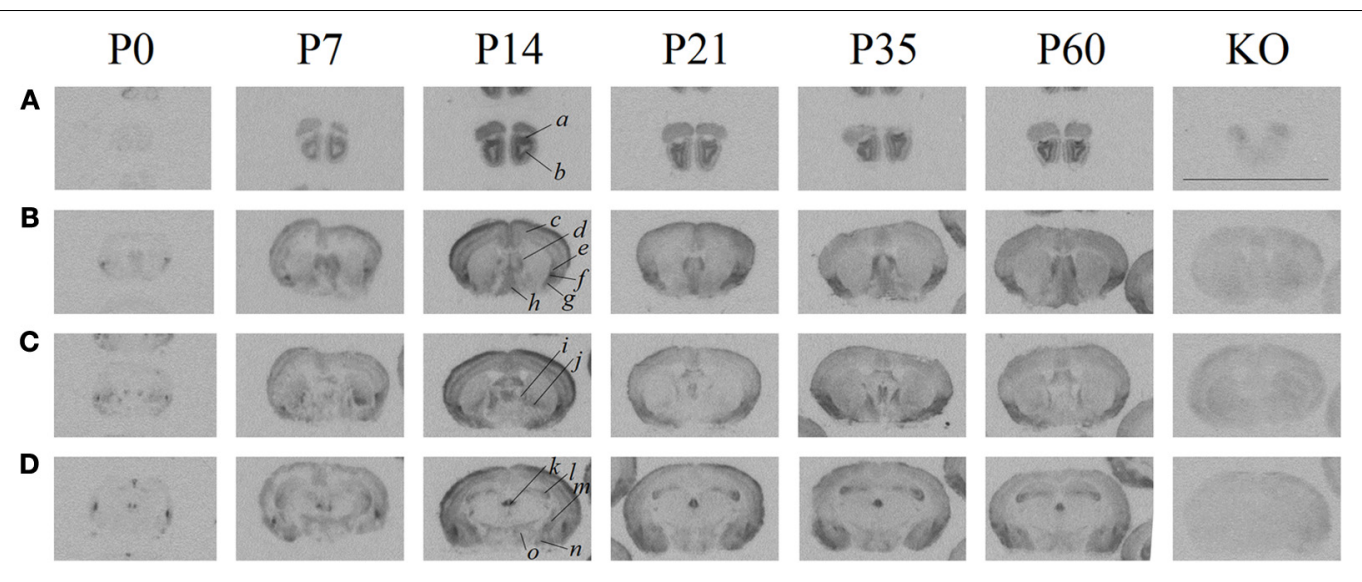

FIGURE 1 | Receptor autoradiography in C57BL/6J mice at several post-natal ages and coronal levels reveals brain areas of OXTR ligand binding, and the lack of specific OXTR ligand binding in OXTR KO brain assessed at P60.(A) accessory (a) and main (b) olfactory bulbs (B) neocortex (c), septum (d), claustrum (e), endopiriform cortex (f), piriform cortex (g), diagonal band of Broca (h), (C) bed nucleus of the stria terminalis (i), ventral caudatoputamen (j), (D) periventricular thalamus ( $k$ ), CA3 hippocampus (I), central amygdala $(m)$, medial amygdala $(n)$, hypothalamus (o). Scale bar $=1 \mathrm{~cm}$. 

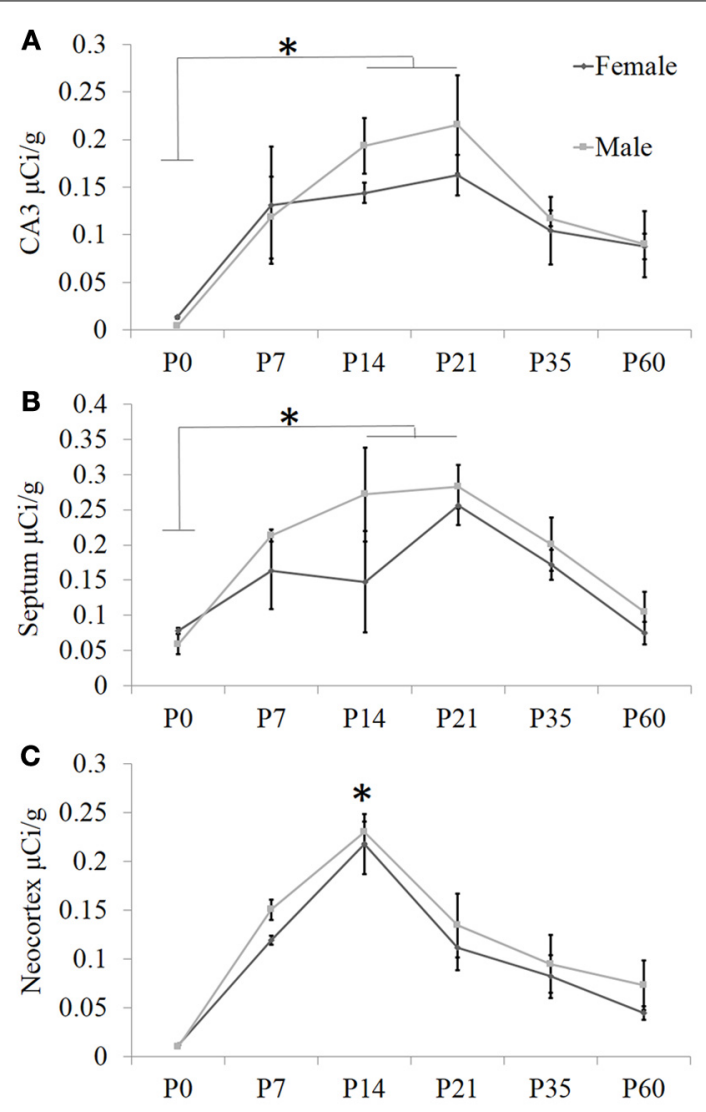

FIGURE 2 | Quantification of receptor autoradiography for OXTR in C57BL/6J mice demonstrates transient developmental profiles. OXTR binding with highly selective OXTR ligand is evident in (A) the CA3 of the dorsal hippocampus, (B) the septum, and (C) the neocortex sampled at S1. For all three brain regions, there was a main effect of age. In the septum and the hippocampus, this was driven by the difference in binding between P0 and P14-P21. In the neocortex, the main effect of age was stronger and driven substantially by the peak at P14. ${ }^{*} p<0.05$.

26.24, $p<0.0001]$, and no sex $\times$ age interaction $\left[F_{(5,24)}=0.19\right.$, $p=0.96]$. Bonferroni-corrected post-hoc tests indicate that for the septum and the hippocampus, the P0 time-point was significantly different from the peak between P14 and P21 $(p<0.05)$. However, post-hoc tests for the neocortex showed that P14 was a significant peak of ligand binding which differed statistically from earlier (P0) and later (P35, P60) time points $(p<0.05)$. Thus, there are distinct regional patterns of differential OXTR ligand binding during postnatal development.

Based on these data, we performed a secondary, layerspecific analysis of the developing neocortex. Post-processed neuroanatomical landmarks of the same tissue used in the receptor autoradiography facilitated localization of OXTR to layers II/III in the pre-weaning neocortex (Figure 3). We quantified OXTR binding in specific layers (II/III, IV, V, VI) across the neocortex in S1. As with the data from combined neocortical layers, there was no main effect of sex $\left[F_{(1,111)}=0.58, p=0.45\right]$, a significant main effect of age $\left[F_{(5,111)}=141.31, p<0.001\right]$, and as expected in this analysis, a significant main effect of neocortical layer $\left[F_{(3,111)}=117.90, p<0.001\right]$. There was also a significant age $\times$ layer interaction $\left[F_{(15,111)}=24.21, p<0.001\right]$ evident in the peak binding of OXTR in layer II/III at P14. Bonferroni corrected post-hoc tests indicated that OXTR ligand binding density in layer II/III at P14 was significantly greater than all other ages and layers examined $(p<0.05)$.

In addition to modest ligand binding in the forebrain, hindbrain, and spinal cord, we observed OXTR ligand binding in several tissues throughout the E18.5 embryo (Figure 4). Dense binding was evident in a very limited number of peripheral tissues, including the dermis, brown fat, adrenal gland, kidney, genitourinary tract, testes (not shown), the olfactory sensory epithelium, and the oral cavity including the tongue, palate, and the anterior mandible.

\section{DISCUSSION}

The present study demonstrates an unexpected, transient developmental profile of OXTR ligand binding throughout the neocortex in the developing mouse that is in part different from other species examined previously. These data have direct implications for the interpretation of functional data from mice, as the major model of genetic perturbation of OXTR signaling. Other groups have previously mapped OXTR in developing rodents, using this ligand. Tribollet et al. (1989) and Shapiro and Insel (1989) described the postnatal development of OXTR ligand binding in the rat. They observed continuously high levels of binding in the accessory olfactory bulb, the ventral subiculum, and the central amygdala, which is similar to what we observe in mice. Unlike mice, however, rats show persistent binding in the dorsal caudate-putamen; mice do not show evidence of specific binding in this region at any age. Also unlike mice, rats exhibit transient binding in the dorsal subiculum and anteroventral thalamus. As previously described (Shapiro and Insel, 1989; Tribollet et al., 1989), rats showed dramatic transient OXTR ligand binding in the neocortex, but the expression was limited to the midline cingulate neocortex. The data in rats contrast with our findings in mice, which demonstrate potential for a broader neocortical role for OXTR outside of limbic neocortex. Wang and Young (1997) mapped OXTR with this ligand in postnatal vole brains, with a particular focus on the septum, although the images in that paper demonstrate transient (although not quantified) postnatal OXTR binding throughout the neocortex of voles as well. The unique and species-specific developmental profiles of OXTR distribution patterns allow for a potential neural substrate of species differences in OXT-dependent developmental trajectories. Perhaps as in adults (Insel and Shapiro, 1992; Young et al., 1996a; Young, 1999; Campbell et al., 2009; Ophir et al., 2012), species differences in OXTR distribution patterns in the developing brain may give rise to important species differences in maturation of social behaviors. Despite the variations in expression patterns of OXTR, rodent species in general are consistent in the timing of the transient developmental peak- neocortical OXTR peaks in the 2nd and 3rd postnatal week, paralleling the major time period of synaptic wiring and pruning in subcortical and cortical forebrain regions (Levitt, 2003; Li et al., 2010). This may provide some clues regarding developmental sensitive periods for the early role 

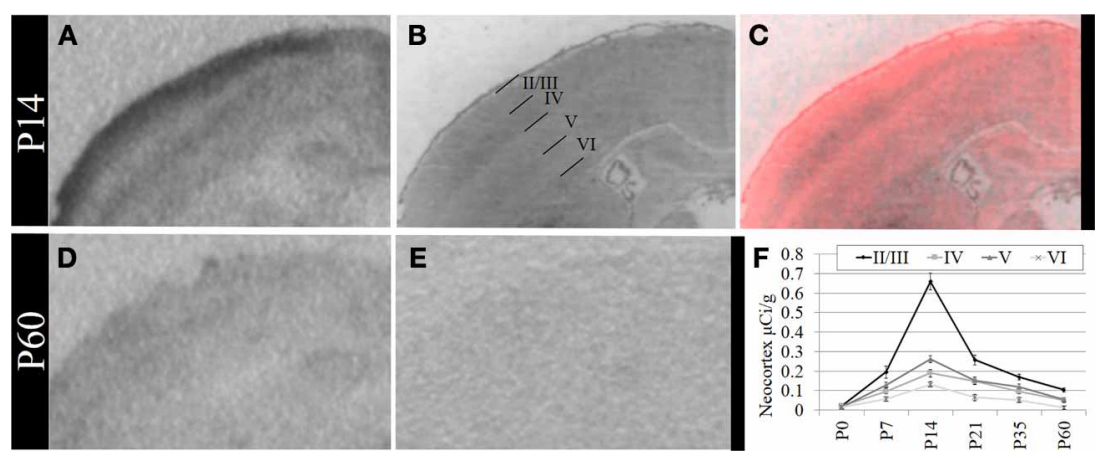

FIGURE 3 | OXTR ligand binding in the neocortex is prominent in layer II/III. (A) OXTR is abundant in neocortical layers II/III at P14. (B) Nissl counterstain of section in (A). (C) OXTR is pseudo-colored red in a composite image of panels (A) and (B), which indicates that OXTR is prominent in layers II/III but not in layer IV or VI. OXTR is present in upper layer V. There is significantly reduced neocortical OXTR in P60 mice (D) which are only slightly above tissue background compared to OXTR KO P60 neocortex (E). (F) Quantification of OXTR binding demonstrates the transient ligand binding of OXTR in upper layers across post-natal development.

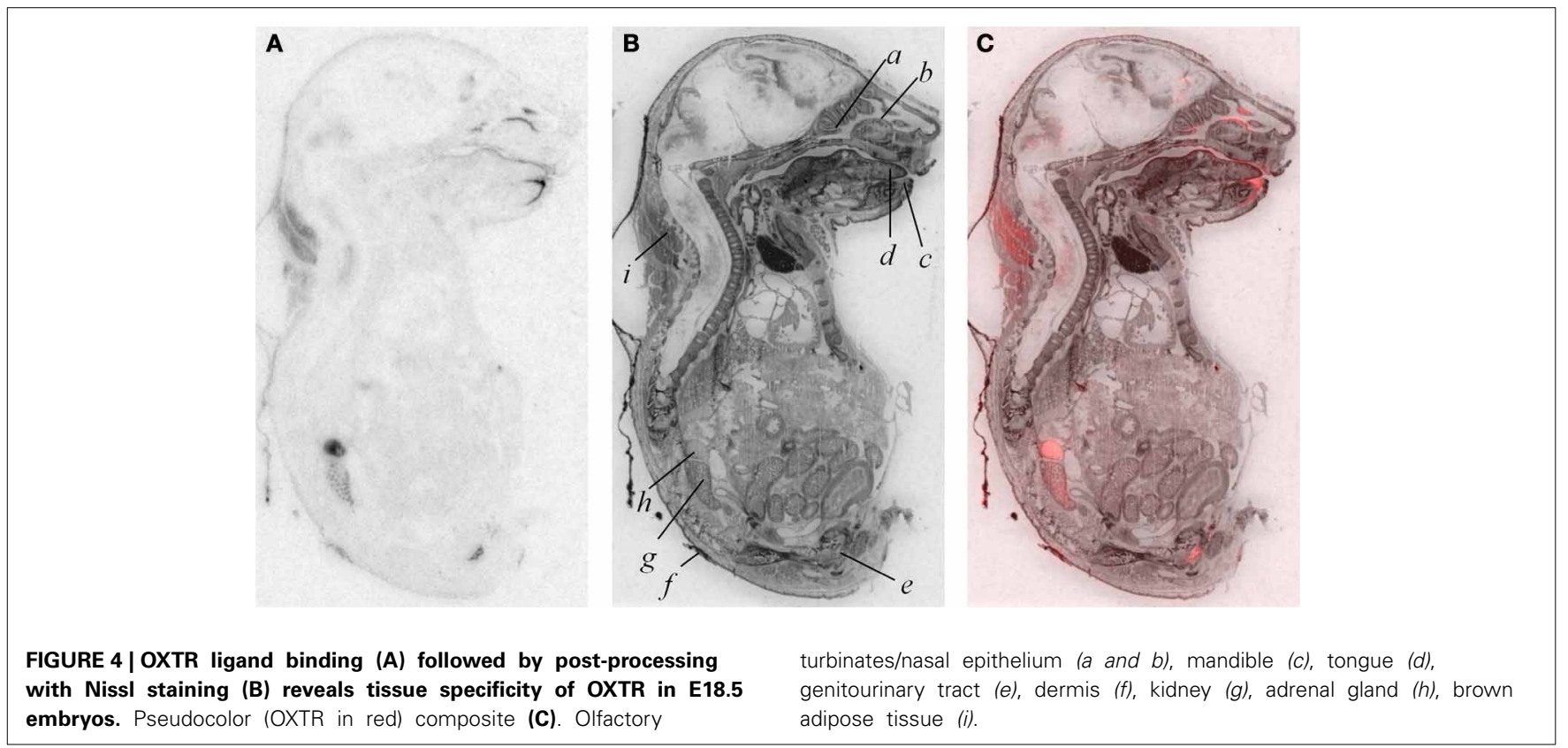

of OXTR in the ontogeny of circuits that mediate behavioral development.

In rats, Tribollet et al. (1989) defined two areas with postpubertal onset of ligand binding: the ventromedial nucleus of the hypothalamus and the islands of Calleja of the olfactory tubercle. While we detected some ligand binding in both of these areas of mice, we were unable to detect a similar post-pubertal onset in these areas or any other related structures (data not shown). This is likely due to an actual lack of adult onset of ligand binding in mice. While unlikely, it is possible that there are technical differences in sampling through these nuclei, which would have missed limited regions of expression, due to the smaller brain size of the mouse.

Our discovery of transient OXTR throughout the developing mouse neocortex permits additional interpretations of data from Sala et al. (2011), who report that the OXTR KO mouse has lower seizure thresholds and impaired cognitive flexibility in the reversal of the T maze. The authors conclude that perhaps the loss of OXTR in the adult hippocampus is the mechanism responsible for the lower seizure thresholds. An alternative interpretation is that developmentally transient neocortical OXTR expression influences experience-dependent neocortical development that may be disrupted in the OXTR KO mouse, contributing to lowered seizure thresholds. Orphan data from the initial reports of the OXT KO mouse support this hypothesis: there was robust c-Fos activation present in S1 neocortex of the adult OXT KO mouse after exposure to a social stimulus, compared to an absence of c-Fos activation in the adult WT mouse after similar exposure (Ferguson et al., 2001). Because there are very few OXTR receptors in the adult neocortex, it is possible that this activation difference in adult OXT KO reflects atypical or labored neural processing of social information after a developmental trajectory 
that could not use OXT signals to shape experience-dependent neocortical development. As observed in the hippocampus (Sala et al., 2011; Owen et al., 2013) and the infralimbic medial prefrontal cortex (Ninan, 2011), OXTR may contribute to signal-tonoise processing throughout the entire neocortex by regulating excitatory and inhibitory balance during post-natal development. The hypothesis that OXT, via OXTR, shapes the experiencedependent plasticity of the entire neocortex during the onset of multisensory integration could be tested using conditional mouse lines in which gene expression would be manipulated with temporal specificity.

Given some of the developmental differences in OXTR across species, there are challenges in determining which animal models provide the most relevant translation to understand the role of OXT signaling in human brain development. Resolving this dilemma becomes especially important with the increased momentum to understand a potential therapeutic role for OXT in autism spectrum and other neurodevelopmental disorders (Bartz and Hollander, 2008; Modi and Young, 2012; Miller, 2013). While a combination of approaches are most likely to yield insight, descriptive data from BrainSpan Allen Institute gene expression data set and from Kang et al. (2011) reveal a peak of OXTR mRNA expression in multiple neocortical regions in postmortem tissue from neonates and infants, with lower levels prenatally and in adolescents and adults. This developmental epoch corresponds to high levels of experience-dependent plasticity for the acquisition of face discrimination (Pascalis et al., 2002, 2005) and language recognition (Kuhl, 2004), as well as peak synaptogenesis (Huttenlocher and Dabholkar, 1997). Perhaps developmental mouse models of OXT system function will be valuable for elucidating a role for OXT in mammalian experience-dependent neocortical development.

A by-product of our developmental study was the generation of unique data prenatally at E18.5, a time proximate to the transition to parturition. Parturition is an important developmental time-point with exposure to high levels of maternal OXT (Kuwabara et al., 1987; Douglas et al., 2002; Tyzio et al., 2006; Khazipov et al., 2008; Mazzuca et al., 2011). Prenatal binding of this OXTR ligand has not been examined in the whole embryo. We observed dense OXTR ligand binding in several embryonic tissues, involved in the regulation of homeostasis and are of great interest in the potential mechanisms of the transition to postnatal life.

These whole embryo OXTR binding data lead to testable novel hypotheses. One testable hypothesis would be to consider the possibility that OXTR in the developing adrenal gland contributes to the stress hyporesponsive period (SHRP)- the period of perinatal development, during which time adrenal activity is suppressed including reduced adrenal sensitivity to ACTH (Rosenfeld et al., 1991) (i.e., less corticosterone release). If OXTR contributed to the SHRP, we would expect a developmental regulation of the levels or function of OXTR in the adrenal gland, with changes in adrenal OXTR function around post-natal day 12-14.

We also observed robust OXTR ligand binding in the oral cavity in near-term embryos, which has not been described previously. In the adult mouse, OXTR has been identified on a subset of glial-like type I taste cells, and in some cells on the periphery of taste buds, where OXT can elicit calcium signals (Sinclair et al., 2010). Our embryo data suggest the following testable hypothesis: OXT present in either amniotic fluid during parturition or in breast milk (Takeda et al., 1986) could activate these receptors to initiate a cascade of signals to help orient the newborn to maternal cues.

OXTR in specific peripheral tissues of the term embryo may facilitate a physiologically coordinated transition to postnatal life. We do not yet know if these peripheral tissues expressing the receptor at E18.5 continue to express OXTR at the same levels, or if there are developmental changes in OXTR in these areas. The data observed in the postnatal brain presented here suggest that OXTR in the neocortex is well-positioned to play a role in the experience-dependent developmental plasticity of the neocortex. These are tractable hypotheses that may lead to a far more detailed understanding of the mechanisms through which OXTR signaling may orchestrate the transition to postnatal life and the developmental emergence of a species-relevant repertoire of social and/or OXT-modulated behaviors (Hammock and Levitt, 2006).

\section{AUTHOR CONTRIBUTIONS}

Elizabeth A. D. Hammock designed and performed the ligand binding studies and data analysis, and Elizabeth A. D. Hammock and Pat Levitt prepared the manuscript.

\section{ACKNOWLEDGMENTS}

These studies and manuscript preparation were funded by NIH MH080759 to Pat Levitt and a NARSAD Young Investigator Award to Elizabeth A. D. Hammock.

\section{REFERENCES}

Allen Brain Atlas Resources [Internet]. Seattle (WA): Allen Institute for Brain Science. (C2009. Available online at: http://www.brain-map.org [Online]. [Accessed].

Bakermans-Kranenburg, M. J., and van Ijzendoorn, M. H. (2013). A sociability gene? Meta-analysis of oxytocin receptor genotype effects in humans. Psychiatr. Genet. doi: 10.1097/YPG.0b013e3283643684. [Epub ahead of print].

Bartz, J. A., and Hollander, E. (2008). Oxytocin and experimental therapeutics in autism spectrum disorders. Prog. Brain Res. 170, 451-462. doi: 10.1016/S00796123(08)00435-4

BrainSpan Atlas of the Developing Human Brain Website: (C2012 Allen Institute for Brain Science. BrainSpan Atlas of the Developing Human Brain [Internet]. Available online at: http://brainspan.org/ [Online]. [Accessed].

Campbell, P., Ophir, A. G., and Phelps, S. M. (2009). Central vasopressin and oxytocin receptor distributions in two species of singing mice. J. Comp. Neurol. 516, 321-333. doi: 10.1002/cne.22116

Catania, E. H., Pimenta, A., and Levitt, P. (2008). Genetic deletion of Lsamp causes exaggerated behavioral activation in novel environments. Behav. Brain Res. 188, 380-390. doi: 10.1016/j.bbr.2007.11.022

Douglas, A. J., Leng, G., and Russell, J. A. (2002). The importance of oxytocin mechanisms in the control of mouse parturition. Reproduction 123, 543-552. doi: 10.1530/rep.0.1230543

Elands, J., Beetsma, A., Barberis, C., and de Kloet, E. R. (1988). Topography of the oxytocin receptor system in rat brain: an autoradiographical study with a selective radioiodinated oxytocin antagonist. J. Chem. Neuroanat. 1, 293-302.

Ferguson, J. N., Aldag, J. M., Insel, T. R., and Young, L. J. (2001). Oxytocin in the medial amygdala is essential for social recognition in the mouse. J. Neurosci. 21, 8278-8285.

Ferguson, J. N., Young, L. J., Hearn, E. F., Matzuk, M. M., Insel, T. R., and Winslow, J. T. (2000). Social amnesia in mice lacking the oxytocin gene. Nat. Genet. 25, 284-288. doi: $10.1038 / 77040$ 
GENSAT. (2003). The Gene Expression Nervous System Atlas (GENSAT) Project, NINDS Contracts N01NS02331 \& HHSN271200723701C to The Rockefeller University (New York, NY).

Gong, S., Zheng, C., Doughty, M. L., Losos, K., Didkovsky, N., Schambra, U. B., et al. (2003). A gene expression atlas of the central nervous system based on bacterial artificial chromosomes. Nature 425, 917-925. doi: 10.1038/nature02033

Gould, B. R., and Zingg, H. H. (2003). Mapping oxytocin receptor gene expression in the mouse brain and mammary gland using an oxytocin receptor-LacZ reporter mouse. Neuroscience 122, 155-167. doi: 10.1016/S03064522(03)00283-5

Gross, C., Zhuang, X., Stark, K., Ramboz, S., Oosting, R., Kirby, L., et al. (2002). Serotonin1A receptor acts during development to establish normal anxiety-like behaviour in the adult. Nature 416, 396-400. doi: 10.1038/416396a

Guastella, A. J., and Macleod, C. (2012). A critical review of the influence of oxytocin nasal spray on social cognition in humans: evidence and future directions. Horm. Behav. 61, 410-418. doi: 10.1016/j.yhbeh.2012.01.002

Hammock, E. A., and Levitt, P. (2012). Modulation of parvalbumin interneuron number by developmentally transient neocortical vasopressin receptor 1a (VlaR). Neuroscience 222C, 20-28. doi: 10.1016/j.neuroscience.2012.07.025

Hammock, E. A. D., and Levitt, P. (2006). The discipline of neurobehavioral development: the emerging interface of processes that build circuits and skills. Hum. Dev. 49, 294-309. doi: 10.1159/000095581

Huttenlocher, P. R., and Dabholkar, A. S. (1997). Regional differences in synaptogenesis in human cerebral cortex. J. Comp. Neurol. 387, 167-178. doi: 10.1002/(SICI) 1096-9861(19971020)387:2 < 167::AID-CNE1 > 3.0.CO;2-Z

Insel, T. R., and Shapiro, L. E. (1992). Oxytocin receptor distribution reflects social organization in monogamous and polygamous voles. Proc. Natl. Acad. Sci. U.S.A. 89, 5981-5985. doi: 10.1073/pnas.89.13.5981

Insel, T. R., Young, L., Witt, D. M., and Crews, D. (1993). Gonadal steroids have paradoxical effects on brain oxytocin receptors. J. Neuroendocrinol. 5, 619-628. doi: 10.1111/j.1365-2826.1993.tb00531.x

Jimenez, A., Fernandez, R., Madrid-Bury, N., Moreira, P. N., Borque, C., Pintado, B., et al. (2003). Experimental demonstration that pre- and post-conceptional mechanisms influence sex ratio in mouse embryos. Mol. Reprod. Dev. 66, 162-165. doi: 10.1002/mrd.10345

Jin, D., Liu, H. X., Hirai, H., Torashima, T., Nagai, T., Lopatina, O., et al. (2007). CD38 is critical for social behaviour by regulating oxytocin secretion. Nature 446, 41-45. doi: 10.1038/nature05526

Kang, H. J., Kawasawa, Y. I., Cheng, F., Zhu, Y., Xu, X., Li, M., et al. (2011). Spatio-temporal transcriptome of the human brain. Nature 478, 483-489. doi: $10.1038 /$ nature 10523

Kato, I., Yamamoto, Y., Fujimura, M., Noguchi, N., Takasawa, S., and Okamoto, H. (1999). CD38 disruption impairs glucose-induced increases in cyclic ADPribose, [Ca2+]i, and insulin secretion. J. Biol. Chem. 274, 1869-1872. doi: $10.1074 / \mathrm{jbc} .274 .4 .1869$

Khazipov, R., Tyzio, R., and Ben-Ari, Y. (2008). Effects of oxytocin on GABA signalling in the foetal brain during delivery. Prog. Brain Res. 170, 243-257. doi: $10.1016 / 50079-6123(08) 00421-4$

Kuhl, P. K. (2004). Early language acquisition: cracking the speech code. Nat. Rev. Neurosci. 5, 831-843. doi: 10.1038/nrn1533

Kuwabara, Y., Takeda, S., Mizuno, M., and Sakamoto, S. (1987). Oxytocin levels in maternal and fetal plasma, amniotic fluid, and neonatal plasma and urine. Arch. Gynecol. Obstet. 241, 13-23. doi: 10.1007/BF00931436

Lee, H. J., Caldwell, H. K., Macbeth, A. H., Tolu, S. G., and Young, W. S. 3rd. (2008a). A conditional knockout mouse line of the oxytocin receptor. Endocrinology 149, 3256-3263. doi: 10.1210/en.2007-1710

Lee, H. J., Caldwell, H. K., Macbeth, A. H., and Young, W. S. 3rd. (2008b). Behavioural studies using temporal and spatial inactivation of the oxytocin receptor. Prog. Brain Res. 170, 73-77. doi: 10.1016/S0079-6123(08) 00407-X

Levitt, P. (2003). Structural and functional maturation of the developing primate brain. J. Pediatr. 143, S35-S45. doi: 10.1067/S0022-3476(03)00400-1

Li, M., Cui, Z., Niu, Y., Liu, B., Fan, W., Yu, D., et al. (2010). Synaptogenesis in the developing mouse visual cortex. Brain Res. Bull. 81, 107-113. doi: 10.1016/j.brainresbull.2009.08.028

Lim, M., Hammock, E., and Young, L. (2004). A method for acetylcholinesterase staining of brain sections previously processed for receptor autoradiography. Biotech. Histochem. 79, 11-16. doi: 10.1080/10520290410001671344
Macbeth, A. H., Lee, H. J., Edds, J., and Young, W. S. 3rd. (2009). Oxytocin and the oxytocin receptor underlie intrastrain, but not interstrain, social recognition. Genes Brain Behav. 8, 558-567. doi: 10.1111/j.1601-183X.2009.00506.x

Mazzuca, M., Minlebaev, M., Shakirzyanova, A., Tyzio, R., Taccola, G., Janackova, S., et al. (2011). Newborn Analgesia mediated by Oxytocin during delivery. Front. Cell. Neurosci. 5:3. doi: 10.3389/fncel.2011.00003

Miller, G. (2013). Neuroscience. The promise and perils of oxytocin. Science 339, 267-269. doi: 10.1126/science.339.6117.267

Miller, J. A., and Zahniser, N. R. (1987). The use of 14C-labeled tissue paste standards for the calibration of 125I-labeled ligands in quantitative autoradiography. Neurosci. Lett. 81, 345-350. doi: 10.1016/0304-3940(87)90408-3

Modi, M. E., and Young, L. J. (2012). The oxytocin system in drug discovery for autism: animal models and novel therapeutic strategies. Horm. Behav. 61, 340-350. doi: 10.1016/j.yhbeh.2011.12.010

Ninan, I. (2011). Oxytocin suppresses basal glutamatergic transmission but facilitates activity-dependent synaptic potentiation in the medial prefrontal cortex. J. Neurochem. 119, 324-331. doi: 10.1111/j.1471-4159.2011.07430.x

Nishimori, K., Young, L. J., Guo, Q., Wang, Z., Insel, T. R., and Matzuk, M. M. (1996). Oxytocin is required for nursing but is not essential for parturition or reproductive behavior. Proc. Natl. Acad. Sci. U.S.A. 93, 11699-11704. doi: 10.1073/pnas.93.21.11699

Ophir, A. G., Gessel, A., Zheng, D. J., and Phelps, S. M. (2012). Oxytocin receptor density is associated with male mating tactics and social monogamy. Horm. Behav. 61, 445-453. doi: 10.1016/j.yhbeh.2012.01.007

Owen, S. F., Tuncdemir, S. N., Bader, P. L., Tirko, N. N., Fishell, G., and Tsien, R. W. (2013). Oxytocin enhances hippocampal spike transmission by modulating fast-spiking interneurons. Nature 500, 458-462. doi: 10.1038/nature12330

Pagani, J. H., Lee, H. J., and Young, W. S. 3rd. (2011). Postweaning, forebrainspecific perturbation of the oxytocin system impairs fear conditioning. Genes Brain Behav. 10, 710-719. doi: 10.1111/j.1601-183X.2011.00709.x

Pascalis, O., de Haan, M., and Nelson, C. A. (2002). Is face processing speciesspecific during the first year of life? Science 296, 1321-1323. doi: 10.1126/science.1070223

Pascalis, O., Scott, L. S., Kelly, D. J., Shannon, R. W., Nicholson, E., Coleman, M., et al. (2005). Plasticity of face processing in infancy. Proc. Natl. Acad. Sci. U.S.A. 102, 5297-5300. doi: 10.1073/pnas.0406627102

Paxinos, G., and Franklin, K. B. J. (2001). The Mouse Brain in Stereotaxic Coordinates. San Diego, CA: Academic Press.

Pedersen, C. A., Vadlamudi, S. V., Boccia, M. L., and Amico, J. A. (2006). Maternal behavior deficits in nulliparous oxytocin knockout mice. Genes Brain Behav. 5, 274-281. doi: 10.1111/j.1601-183X.2005.00162.x

Rosenfeld, P., Gutierrez, Y. A., Martin, A. M., Mallett, H. A., Alleva, E., and Levine, S. (1991). Maternal regulation of the adrenocortical response in preweanling rats. Physiol. Behav. 50, 661-671. doi: 10.1016/0031-9384(91)90001-5

Sala, M., Braida, D., Lentini, D., Busnelli, M., Bulgheroni, E., Capurro, V., et al. (2011). Pharmacologic rescue of impaired cognitive flexibility, social deficits, increased aggression, and seizure susceptibility in oxytocin receptor null mice: a neurobehavioral model of autism. Biol. Psychiatry 69, 875-882. doi: 10.1016/j.biopsych.2010.12.022

Shapiro, L. E., and Insel, T. R. (1989). Ontogeny of oxytocin receptors in rat forebrain: a quantitative study. Synapse 4, 259-266. doi: 10.1002/syn.890040312

Sinclair, M. S., Perea-Martinez, I., Dvoryanchikov, G., Yoshida, M., Nishimori, K., Roper, S. D., et al. (2010). Oxytocin signaling in mouse taste buds. PLoS ONE 5:e11980. doi: 10.1371/journal.pone.0011980

Snijdewint, F. G., Van Leeuwen, F. W., and Boer, G. J. (1989). Ontogeny of vasopressin and oxytocin binding sites in the brain of Wistar and Brattleboro rats as demonstrated by lightmicroscopical autoradiography. J. Chem. Neuroanat. 2, 3-17.

Takayanagi, Y., Yoshida, M., Bielsky, I. F., Ross, H. E., Kawamata, M., Onaka, T., et al. (2005). Pervasive social deficits, but normal parturition, in oxytocin receptor-deficient mice. Proc. Natl. Acad. Sci. U.S.A. 102, 16096-16101. doi: 10.1073/pnas.0505312102

Takeda, S., Kuwabara, Y., and Mizuno, M. (1986). Concentrations and origin of oxytocin in breast milk. Endocrinol. Jpn. 33, 821-826. doi: 10.1507/endocrj1954.33.821

Thevenaz, P., Ruttimann, U. E., and Unser, M. (1998). A pyramid approach to subpixel registration based on intensity. IEEE Trans. Image Process. 7, 27-41. doi: $10.1109 / 83.650848$ 
Tribollet, E., Charpak, S., Schmidt, A., Dubois-Dauphin, M., and Dreifuss, J. J. (1989). Appearance and transient expression of oxytocin receptors in fetal, infant, and peripubertal rat brain studied by autoradiography and electrophysiology. J. Neurosci. 9, 1764-1773.

Tribollet, E., Dubois-Dauphin, M., Dreifuss, J. J., Barberis, C., and Jard, S. (1992). Oxytocin receptors in the central nervous system. Distribution, development, and species differences. Ann. N.Y. Acad. Sci. 652, 29-38. doi: 10.1111/j.17496632.1992.tb34343.x

Tribollet, E., Goumaz, M., Raggenbass, M., and Dreifuss, J. J. (1991). Appearance and transient expression of vasopressin and oxytocin receptors in the rat brain. J. Recept. Res. 11, 333-346. doi: 10.3109/1079989910 9066412

Tyzio, R., Cossart, R., Khalilov, I., Minlebaev, M., Hubner, C. A., Represa, A., et al. (2006). Maternal oxytocin triggers a transient inhibitory switch in GABA signaling in the fetal brain during delivery. Science 314, 1788-1792. doi: $10.1126 /$ science. 1133212

Wang, Z., and Young, L. J. (1997). Ontogeny of oxytocin and vasopressin receptor binding in the lateral septum in prairie and montane voles. Brain Res. Dev. Brain Res. 104, 191-195. doi: 10.1016/S0165-3806(97)00138-7

Yoshida, M., Takayanagi, Y., Inoue, K., Kimura, T., Young, L. J., Onaka, T., et al. (2009). Evidence that oxytocin exerts anxiolytic effects via oxytocin receptor expressed in serotonergic neurons in mice. J. Neurosci. 29, 2259-2271. doi: 10.1523/JNEUROSCI.5593-08.2009

Young, L. J. (1999). Frank A. Beach Award. Oxytocin and vasopressin receptors and species-typical social behaviors. Horm. Behav. 36, 212-221. doi: 10.1006/hbeh.1999.1548

Young, L. J., Huot, B., Nilsen, R., Wang, Z., and Insel, T. R. (1996a). Species differences in central oxytocin receptor gene expression: comparative analysis of promoter sequences. J. Neuroendocrinol. 8, 777-783. doi: 10.1046/j.13652826.1996.05188.x

Young, W. S. 3rd., Shepard, E., Amico, J., Hennighausen, L., Wagner, K. U., Lamarca, M. E., et al. (1996b). Deficiency in mouse oxytocin prevents milk ejection, but not fertility or parturition. J. Neuroendocrinol. 8, 847-853. doi: 10.1046/j.1365-2826.1996.05266.x

Zhang, B. J., Kusano, K., Zerfas, P., Iacangelo, A., Young, W. S. 3rd., and Gainer, H. (2002). Targeting of green fluorescent protein to secretory granules in oxytocin magnocellular neurons and its secretion from neurohypophysial nerve terminals in transgenic mice. Endocrinology 143, 1036-1046. doi: 10.1210/en.143.3.1036

Conflict of Interest Statement: The authors declare that the research was conducted in the absence of any commercial or financial relationships that could be construed as a potential conflict of interest.

Received: 18 September 2013; paper pending published: 15 October 2013; accepted: 22 November 2013; published online: 11 December 2013.

Citation: Hammock EAD and Levitt P (2013) Oxytocin receptor ligand binding in embryonic tissue and postnatal brain development of the C57BL/6J mouse. Front. Behav. Neurosci. 7:195. doi: 10.3389/fnbeh.2013.00195

This article was submitted to the journal Frontiers in Behavioral Neuroscience. Copyright (c) 2013 Hammock and Levitt. This is an open-access article distributed under the terms of the Creative Commons Attribution License (CC BY). The use, distribution or reproduction in other forums is permitted, provided the original author(s) or licensor are credited and that the original publication in this journal is cited, in accordance with accepted academic practice. No use, distribution or reproduction is permitted which does not comply with these terms. 